\title{
Adaptive Robust Controller Designs Applied to Free-Floating Space Manipulators in Task Space
}

\author{
Tatiana Pazelli, Marco Terra \\ and Adriano Siqueira \\ University of São Paulo \\ São Carlos, São Paulo \\ Brazil
}

\section{Introduction}

Space robots are featured by a dynamic coupling which causes the rotation of the main body with the coordinated motions of the arm. A number of dynamic and control problems are unique to this area due to the distinctive and complex dynamics found in many applications. In-space operations such as assembly, inspection and maintenance of satellites or space stations have been receiving considerable research efforts. Considering the hostile environment where a space robot operates, which can deteriorate its structure and physical characteristics, and also considering the difficulty of taking the system back to reformulate its dynamic model due to these uncertainties, the proposal of intelligent adaptive robust controllers to this kind of system becomes very interesting.

One of the representative types of space robotic systems identified by [Dubowsky \& Papadopoulos (1993)], free-floating space manipulators are systems that allows the spacecraft to move freely in response to the manipulator motions in order to conserve fuel and electrical power, [Papadopoulos \& Dubowsky (1991)]. Trajectory planning algorithms have been developed in order to minimize the reaction motion of the free-floating base while executing the manipulator task, [Huang \& Xu (2006); Liu et al. (2009); Papadopoulos et al. (2005); Torres \& Dubowsky (1992); Tortopidis \& Papadopoulos (2006)]. In case of redundant manipulators, coordinated spacecraft/manipulator motion control has been addressed in [Caccavale \& Siciliano (2001); Dubowsky \& Torres (1991)].

Solving control problems in joint space is an inconvenient task for a space robot with a freefloating base. When the base is free-floating, the kinematic mapping from task space to joint space, where the control is executed, becomes non-unique because of non-integrable angular momentum conservation. This may cause non-existence of the reference trajectory in joint space. Also, parametric uncertainties appear not only in the dynamic equation, but also in kinematic mapping from the joint space to the task space due to the absence of a fixed base. The model inaccuracies lead to the deviation of operational space trajectory provided by the kinematic mapping. [Parlaktuna \& Ozkan (2004)] and [Abiko \& Hirzinger (2009)] applied on-line adaptive techniques to deal with parametric uncertainties in controlling free-floating 
manipulators at task space. [Fu et al. (2007)], on the other hand, established an off-line adaptive estimator to provide accurate identified parameters to a dynamic control law. In order to cope with the nonlinear parameterization problem of the dynamic model of the free-floating space robot system, [Gu \& Xu (1993)], [Parlaktuna \& Ozkan (2004)] and [Fu et al. (2007)] have modeled the system as an extended arm, and [Abiko \& Hirzinger (2009)] used the inverted chain approach to explicitly describe the coupled dynamics between the end-effector and the robot arm.

This chapter deals with the problem of robust trajectory tracking control in task space for free-floating manipulator systems subject to plant uncertainties and external disturbances. To conduct a comparative study, adaptive techniques are developed considering nonlinear $\mathcal{H}_{\infty}$ controllers based on game theory. A first approach is proposed considering a well defined structure for the plant, where the parameter uncertainties are represented as external disturbances. Artificial neural networks are applied in two other approaches. The first one applies the intelligent system to learn the dynamic behavior of the robotic system, which is considered totally unknown. The second intelligent strategy considers a well defined nominal model structure and the neural networks are applied to estimate only the behavior of parametric uncertainties and the spacecraft dynamics, considered here as non-modeled dynamics. The $\mathcal{H}_{\infty}$ criterion is applied to the proposed techniques to attenuate the effect of estimation errors and external disturbances.

The dynamic model of the free-floating space manipulator (SM) is described in this chapter through the Dynamically Equivalent Manipulator (DEM) approach, [Liang et al. (1996)]. The DEM is a fixed-base manipulator with its first joint being a passive spherical one and, whose model is both kinematically and dynamically equivalent to the SM dynamics.

Trajectory tracking of the SM's end-effector in task space is considered for simulation. A fixedbase, three-link, planar manipulator whose first joint is configured as passive (UArmII - Underactuated Arm II) is taken as a dynamically equivalent reference to a free-floating space planar manipulator with two links. Parametric uncertainties and finite energy exogenous disturbances are included in the nominal model. In order to validate and compare the proposed strategies, graphical and numerical analysis are provided.

The chapter is organized as follows: Section 2 presents the model description through the DEM approach; the solutions for the nonlinear $\mathcal{H}_{\infty}$ control problems based on model and neural networks are presented in Section 3; and, finally, simulation results for a two-link freefloating space manipulator are presented in Section 4.

\section{Model Description and Problem Formulation}

\subsection{Free-Floating Space Manipulator Mapped by a Dynamically Equivalent Fixed-Base Ma- nipulator}

Consider an $n$-link serial-chain rigid manipulator mounted on a free-floating base and that no external forces and torques are applied on this system. Consider also the Dynamically Equivalent Manipulator (DEM) approach, [Liang et al. (1996)]. The DEM is an $(n+1)$-link fixed-base manipulator with its first joint being a passive spherical one and whose model is both kinematically and dynamically equivalent to the SM dynamics. Since it is a conventional manipulator, it can be physically built and experimentally used to study control algorithms for space manipulators.

Figure 1 shows the representation and the parameter notation for both SM and DEM manipulators. Let the SM parameters be identified by apostrophe $\left(\phi^{\prime}, \theta^{\prime}, \rho^{\prime}, J^{\prime}\right)$, the links of the manipulators are numbered from 2 to $n+1$; the Z-Y-Z euler angles $(\phi, \theta, \rho)$ represent the SM 


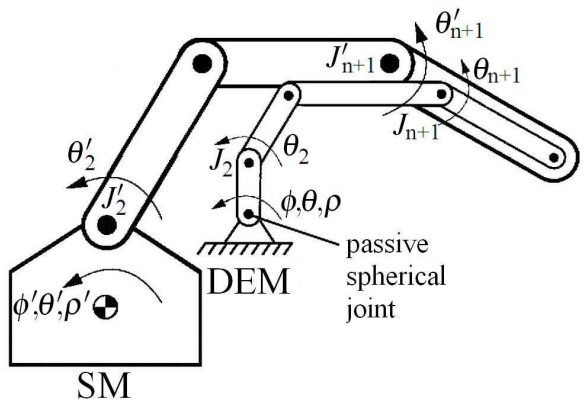

Fig. 1. The space manipulator and its corresponding DEM.

base attitude and the DEM first passive joint orientation; $J_{i}$ is the joint connecting the $(i-1)$-th link and $i$-th link; $\theta_{i}$ is the rotation of the $i$-th link around joint $J_{i} ; C_{i}$ is the center of mass of the $i$-th link; $L_{i}$ is the vector connecting $J_{i}{ }^{\prime}$ and $C_{i}{ }^{\prime} ; R_{i}$ is the vector connecting $C_{i}{ }^{\prime}$ and $J_{i+1}{ }^{\prime} ; l_{c i}$ is the vector connecting $J_{i}$ and $C_{i}$; and $W_{i}$ is the vector connecting $J_{i}$ and $J_{i+1}$.

Considering that the DEM operates in the absence of gravity and that its base is located at the center of mass of the SM, the kinematic and dynamical parameters of the DEM can be found from the SM parameters as

$$
\begin{aligned}
& m_{i}=\frac{M_{t}^{2} m_{i}{ }^{\prime}}{\sum_{k=1}^{i-1} m_{k^{\prime}} \sum_{k=1}^{i} m_{k}{ }^{\prime}}, \\
& i=2, \ldots, n+1 \text {, } \\
& I_{i}=I_{i}^{\prime}{ }^{\prime} \text {, } \\
& i=1, \ldots, n+1, \\
& W_{1}=R_{1} \frac{\sum_{k=1}^{i} m_{k}^{\prime}}{M_{t}}, \\
& W_{i}=R_{i} \frac{\sum_{k=1}^{i} m_{k}{ }^{\prime}}{M_{t}}+L_{i} \frac{\sum_{k=1}^{i-1} m_{k}{ }^{\prime}}{M_{t}}, \quad i=2, \ldots, n+1, \\
& l_{c 1}=0 \text {, } \\
& l_{c i}=L_{i} \frac{\sum_{k=1}^{i-1} m_{k}^{\prime}}{M_{t}},
\end{aligned}
$$

where $M_{t}$ is the total mass of the SM. Observe that the mass of the passive joint, $m_{1}$, is not defined by the equivalence properties.

Let the generalized coordinates $q=\left[\begin{array}{llllll}\phi & \theta & \rho & \theta_{2} & \cdots & \theta_{n+1}\end{array}\right]^{T}$ be partitioned as $q=$ $\left[\begin{array}{ll}q_{b}^{T} & q_{m}^{T}\end{array}\right]^{T}$, where the indexes $b$ and $m$ represent the passive spherical joint (base) and the active joints (manipulator), respectively. From Lagrange theory, dynamic equations of the DEM are given by

$$
M\left(q_{m}\right) \ddot{q}+C\left(q_{m}, \dot{q}\right) \dot{q}=\tau,
$$

where $M\left(q_{m}\right) \in \mathbb{R}^{(n+3 \times n+3)}$ is the symmetric positive definite inertia matrix, $C\left(q_{m}, \dot{q}\right) \in \mathbb{R}^{(n+3 \times n+3)}$ is the matrix of Coriolis and centrifugal forces, and $\tau=$ $\left[\begin{array}{cccccc}0 & 0 & 0 & \tau_{2} & \cdots & \tau_{n+1}\end{array}\right]^{T}$ is the torque vector acting upon the joints of the DEM. Parametric uncertainties can be introduced dividing the parameter matrices $M\left(q_{m}\right)$ and $C\left(q_{m}, \dot{q}\right)$ 
into a nominal and a perturbed part

$$
\begin{aligned}
& M\left(q_{m}\right)=M_{0}\left(q_{m}\right)+\Delta M\left(q_{m}\right), \\
& C\left(q_{m}, \dot{q}\right)=C_{0}\left(q_{m}, \dot{q}\right)+\Delta C\left(q_{m}, \dot{q}\right),
\end{aligned}
$$

where $M_{0}\left(q_{m}\right)$ and $C_{0}\left(q_{m}, \dot{q}\right)$ are nominal matrices and $\Delta M\left(q_{m}\right)$ and $\Delta C\left(q_{m}, \dot{q}\right)$ are the parametric uncertainties.

\subsection{Problem Formulation}

As we are dealing with a free-floating space manipulator, it is considered that only the active joints of the DEM are controlled, with the passive spherical joint not locked. In this case, the passive joint dynamics intervenes with the control of the manipulator active joints.

The vector of orientation and inertial position of the end-effector,

$$
p=\left[\begin{array}{llllll}
\phi_{e f} & \theta_{e f} & \psi_{e f} & x_{e f} & y_{e f} & z_{e f}
\end{array}\right],
$$

is function of free-floating base position and attitude and of generalized coordinates of manipulator joints, $q_{m}$. Once the DEM modelling technique locates the inertial frame origin at the center of mass of the SM, the dependence of end-effector coordinates on base position is eliminated by integrating its equation of linear momentum, [Papadopoulos \& Dubowsky (1991)]. However, the dependence on base attitude cannot be eliminated since the angular momentum of the system cannot be analytically integrated to provide the base attitude as a function of the variables of manipulator joints.

Let $J(q)$ be the Jacobian that relates the velocities of joints coordinates, $\dot{q}$, and the velocities of the end-effector, $\dot{p}$ :

$$
\dot{p}=J(q) \dot{q} .
$$

Considering that $\operatorname{det}(J(q)) \neq 0$, applying (3) and its derivative, $\ddot{p}=\dot{J}(q) \dot{q}+J(q) \ddot{q}$, to (2), we have

$$
\tau=M_{e f}(q) \ddot{p}+C_{e f}(q, \dot{q}) \dot{p},
$$

where

$$
\begin{aligned}
& M_{e f}(q)=M\left(q_{m}\right) J^{-1}(q), \\
& C_{e f}(q, \dot{q})=\left(C\left(q_{m}, \dot{q}\right)-M\left(q_{m}\right) J^{-1}(q) \dot{J}(q, \dot{q})\right) J^{-1}(q) .
\end{aligned}
$$

It must be noted that the Jacobian, $J(q)$, introduces the values of spacecraft's attitude, $q_{b}$, in the dynamic equation matrices, $M_{e f}$ and $C_{e f}$. This does not happen when the problem is formulated in joint space, [Taveira et al. (2006)]. Another remark is that, in this formulation, $M_{e f}(q)$ is not a symmetric positive definite matrix, neither $N_{e f}(q, \dot{q})=\dot{M}_{e f}(q, \dot{q})-2 C_{e f}(q, \dot{q})$ is skew-symmetric. In order to preserve the characteristics of dynamics formulated in joint space, a force transformation is applied to (4), [Lewis et al. (1993)]:

$$
\tau=J^{T}(q) F,
$$

where $F$ is a vector of generalized forces of the end-effector in inertial space. Therefore,

$$
F=\bar{M}_{e f}(q) \ddot{p}+\bar{C}_{e f}(q, \dot{q}) \dot{p},
$$

with

$$
\begin{aligned}
& \bar{M}_{e f}(q)=J^{-T}(q) M_{e f}(q)=J^{-T}(q) M\left(q_{m}\right) J^{-1}(q), \\
& \bar{C}_{e f}(q, \dot{q})=J^{-T}(q) C_{e f}(q, \dot{q})=J^{-T}(q)\left(C\left(q_{m}, \dot{q}\right)-M\left(q_{m}\right) J^{-1}(q) \dot{J}(q, \dot{q})\right) J^{-1}(q) .
\end{aligned}
$$


In this format the dynamic equation formulated in inertial space maintains the structure and properties found in joint space. So, $\bar{M}_{e f}(q)$ is symmetric positive definite and $\bar{N}_{e f}(q, \dot{q})=$ $\dot{\bar{M}}_{e f}(q, \dot{q})-2 \bar{C}_{e f}(q, \dot{q})$ is skew-symmetric.

A characteristic inherited from underactuated manipulators, dealing with a system with $n_{a}$ actuators leads to controlling only $n_{a}$ degrees of freedom at a time, [Siqueira \& Terra (2004)]. The DEM presents $n$ active joints and, then, $n_{a}=n$. So, let's define $p=\left[\begin{array}{ll}p_{u}^{T} & p_{a}^{T}\end{array}\right]^{T}$ the vector of generalized coordinates of the system, with $p_{u} \in \mathbb{R}^{(6-n) \times 1}$ and $p_{a} \in \mathbb{R}^{n \times 1}$, where the indexes $u$ and $a$ represent the passive variables (which are let free during the control procedure) and the controlled variables, respectively. Partitioning equation (6), we have

$$
\left[\begin{array}{c}
F_{u} \\
F_{a}
\end{array}\right]=\left[\begin{array}{cc}
\bar{M}_{e f_{u u}}(q) & \bar{M}_{e f_{u a}}(q) \\
\bar{M}_{e f_{a u}}(q) & \bar{M}_{e f_{a a}}(q)
\end{array}\right]\left[\begin{array}{c}
\ddot{p}_{u} \\
\ddot{p}_{a}
\end{array}\right]+\left[\begin{array}{ll}
\bar{C}_{e f_{u u}}(q, \dot{q}) & \bar{C}_{e f_{u a}}(q, \dot{q}) \\
\bar{C}_{e f_{a u}}(q, \dot{q}) & \bar{C}_{e f_{a a}}(q, \dot{q})
\end{array}\right]\left[\begin{array}{c}
\dot{p}_{u} \\
\dot{p}_{a}
\end{array}\right],
$$

with $\bar{M}_{e f_{u u}} \in \mathbb{R}^{(6-n) \times(6-n)}, \bar{M}_{e f_{u a}} \in \mathbb{R}^{(6-n) \times n}, \bar{M}_{e f_{a u}} \in \mathbb{R}^{n \times(6-n)}, \bar{M}_{e f_{a a}} \in \mathbb{R}^{n \times n}, \bar{C}_{e f_{u u}} \in$ $\mathbb{R}^{(6-n) \times(6-n)}, \bar{C}_{e f_{u a}} \in \mathbb{R}^{(6-n) \times n}, \bar{C}_{e f_{a u}} \in \mathbb{R}^{n \times(6-n)}, \bar{C}_{e f_{a a}} \in \mathbb{R}^{n \times n}, F_{u} \in \mathbb{R}^{(6-n) \times 1}$ e $F_{a} \in \mathbb{R}^{n \times 1}$. This decomposition should also preserve the properties of dynamic equation for the matrices $\bar{M}_{e f_{a a}}(q)$ and $\bar{C}_{e f_{a a}}(q, \dot{q})$ :

- $\bar{M}_{e f_{a a}}(q)=\bar{M}_{e f_{a a}}^{T}(q)>0$ and

- $\bar{N}_{e f_{a a}}(q, \dot{q})=\dot{\bar{M}}_{e f_{a a}}(q, \dot{q})-2 \bar{C}_{e f_{a a}}(q, \dot{q})$ is skew-symmetric.

Define $\delta=\left[\begin{array}{ll}\delta_{u}^{T} & \delta_{a}^{T}\end{array}\right]^{T}$ as a vector representing the sum of parametric uncertainties of the system and $F_{d}$ as a finite energy external disturbance also introduced. Equation (7) can be rewritten as:

$$
\begin{aligned}
{\left[\begin{array}{c}
F_{u} \\
F_{a}
\end{array}\right]+\left[\begin{array}{c}
\delta_{u} \\
\delta_{a}
\end{array}\right]+F_{d}=} & {\left[\begin{array}{cc}
\bar{M}_{e f_{u u}}(q) & \bar{M}_{e f_{u a}}(q) \\
\bar{M}_{e f_{a u}}(q) & \bar{M}_{e f_{a a}}(q)
\end{array}\right]\left[\begin{array}{c}
\ddot{p}_{u} \\
\ddot{p}_{a}
\end{array}\right]+} \\
& +\left[\begin{array}{cc}
\bar{C}_{e f_{u u}}(q, \dot{q}) & \bar{C}_{e f_{u a}}(q, \dot{q}) \\
\bar{C}_{e f_{a u}}(q, \dot{q}) & \bar{C}_{e f_{a a}}(q, \dot{q})
\end{array}\right]\left[\begin{array}{c}
\dot{p}_{u} \\
\dot{p}_{a}
\end{array}\right]
\end{aligned}
$$

where

$$
\left[\begin{array}{c}
\delta_{u}\left(q, \dot{q}, \dot{p}, \ddot{p}, \tau_{d}\right) \\
\delta_{a}\left(q, \dot{q}, \dot{p}, \ddot{p}, \tau_{d}\right)
\end{array}\right]=-\left[\begin{array}{c}
\Delta \bar{M}_{e f_{u u}}(q) \ddot{p}_{u}+\Delta \bar{M}_{e f_{u a}}(q) \ddot{p}_{a}+\Delta \bar{C}_{e f_{u u}}(q, \dot{q}) \dot{p}_{u}+\Delta \bar{C}_{e f_{u a}}(q, \dot{q}) \dot{p}_{a} \\
\Delta \bar{M}_{e f_{a u}}(q) \ddot{p}_{u}+\Delta \bar{M}_{e f_{a a}}(q) \ddot{p}_{a}+\Delta \bar{C}_{e f_{a u}}(q, \dot{q}) \dot{p}_{u}+\Delta \bar{C}_{e f_{a a}}(q, \dot{q}) \dot{p}_{a}
\end{array}\right]
$$

For simplicity of notation, the index 0 referring to the nominal system was suppressed. Let $p_{a}^{d} \in \mathbb{R}^{n}$ and $\dot{p}_{a}^{d} \in \mathbb{R}^{n}$ be the desired reference trajectory and the corresponding velocity for the end-effector controlled variables, respectively. The state tracking error is defined as

$$
\tilde{x}_{e f}=\left[\begin{array}{c}
\dot{p}_{a}-\dot{p}_{a}^{d} \\
p_{a}-p_{a}^{d}
\end{array}\right]=\left[\begin{array}{c}
\dot{\tilde{p}}_{a} \\
\tilde{p}_{a}
\end{array}\right]
$$

The variables $p_{a}^{d}, \dot{p}_{a}^{d}$, and $\ddot{p}_{a}^{d}$ (desired acceleration) are assumed to be within the physical and kinematics limits of the control system and there exists no reference trajectory for the base. Also, assume that $p_{a}^{d}, \dot{p}_{a}^{d}$, and $\ddot{p}_{a}^{d}$, belong entirely to the path independent workspace (PIW), [Torres \& Dubowsky (1992)], and therefore, they will not conduce to any dynamic singularity, i.e., $\operatorname{det}(J) \neq 0$ throughout the path. 
Consider the following state transformation, [Johansson (1990)],

$$
\tilde{z}=T_{0} \tilde{x}_{e f}=\left[\begin{array}{cc}
T_{11} & T_{12} \\
0 & I
\end{array}\right]\left[\begin{array}{c}
\dot{\tilde{p}}_{a} \\
\tilde{p}_{a}
\end{array}\right]
$$

where $T_{11}, T_{12} \in \mathbb{R}^{n \times n}$ are constant matrices to be determined. From (8), (9) and (10), state space representation of the DEM is given by

$$
\begin{aligned}
\dot{\tilde{x}}_{e f} & =\bar{A}_{T_{e f}}(q, \dot{q}) \tilde{x}_{e f}+\bar{B}_{T_{e f}}(q) T_{11}\left(-\bar{F}\left(x_{e_{e f}}\right)-\bar{E}\left(x_{e_{b}}\right)+F_{a}+\delta_{a}+F_{d}\right) \\
& =\bar{A}_{T_{e f}}(q, \dot{q}) \tilde{x}_{e f}+\bar{B}_{T_{e f}}(q) u+\bar{B}_{T_{e f}}(q) \omega,
\end{aligned}
$$

where

$$
\begin{aligned}
& \bar{A}_{T_{e f}}(q, \dot{q})=T_{0}^{-1}\left[\begin{array}{cc}
-\bar{M}_{e f_{a a}}^{-1}(q) \bar{C}_{e f_{a a}}(q, \dot{q}) & 0 \\
I & 0
\end{array}\right] T_{0}, \\
& \bar{B}_{T_{e f}}(q)=T_{0}^{-1}\left[\begin{array}{c}
\bar{M}_{e f_{a a}}^{-1}(q) \\
0
\end{array}\right], \\
& \bar{F}\left(x_{e_{e f}}\right)=\bar{M}_{e f_{a a}}(q)\left(\ddot{p}_{a}^{d}-T_{11}^{-1} T_{12} \dot{\tilde{p}}_{a}\right)+\bar{C}_{e f_{a a}}(q, \dot{q})\left(\dot{p}_{a}^{d}-T_{11}^{-1} T_{12} \tilde{p}_{a}\right), \\
& \bar{E}\left(x_{e_{b}}\right)=\bar{M}_{e f_{a u}}(q) \ddot{p}_{u}+\bar{C}_{e f_{a u}}(q, \dot{q}) \dot{p}_{u}, \\
& u=T_{11}\left(-\bar{F}\left(x_{e_{e f}}\right)-\bar{E}\left(x_{e_{b}}\right)+F_{a}\right), \text { and } \\
& \omega=T_{11}\left(\delta_{a}+F_{d}\right),
\end{aligned}
$$

with $\quad x_{e_{e f}}=\left[\begin{array}{lllllll}q^{T} & \dot{q}^{T} & p_{a}^{T} & \dot{p}_{a}^{T} & \left(p_{a}^{d}\right)^{T} & \left(\dot{p}_{a}^{d}\right)^{T} & \left(\ddot{p}_{a}^{d}\right)^{T}\end{array}\right]^{T}$ and $x_{e_{b}}=$ $\left[\begin{array}{llll}q^{T} & \dot{q}^{T} & \dot{p}_{u}^{T} & \left(\ddot{p}_{u}\right)^{T}\end{array}\right]^{T}$.

The vector of generalized forces related to active variables, $F_{a}$, comes from the second line of equation (7):

$$
F_{a}=\bar{M}_{e f_{a u}}(q) \ddot{p}_{u}+\bar{M}_{e f_{a a}}(q) \ddot{p}_{a}+\bar{C}_{e f_{a u}}(q, \dot{q}) \dot{p}_{u}+\bar{C}_{e f_{a a}}(q, \dot{q}) \dot{p}_{a} .
$$

Decomposing (4) as in (7), the 2nd order non-holonomic constraint imposed by the freefloating base (passive joint at MDE) to the system is described by

$$
M_{e f_{u u}}(q) \ddot{p}_{u}+M_{e f_{u a}}(q) \ddot{p}_{a}+C_{e f_{u u}}(q) \dot{p}_{u}+C_{e f_{u a}}(q) \dot{p}_{a}=0 .
$$

From (12) and (13), we have

$$
F_{a}=M_{F_{a}}(q) \ddot{p}_{a}+C_{F_{a}}(q, \dot{q}) \dot{p},
$$

where

$$
\begin{aligned}
& M_{F_{a}}(q)=\bar{M}_{e f_{a u}}(q)-\bar{M}_{e f_{a u}}(q) M_{e f_{u u}}^{-1}(q) M_{e f_{u a}}(q), \\
& C_{F_{a}}(q, \dot{q})= \\
& {\left[\bar{C}_{e f_{a u}}(q, \dot{q})-\bar{M}_{e f_{a u}}(q) M_{e f_{u u}}^{-1}(q) C_{e f_{u u}}(q, \dot{q}) \quad \bar{C}_{e f_{a a}}(q, \dot{q})-\bar{M}_{e f_{a u}}(q) M_{e f_{u u}}^{-1}(q) C_{e f_{u a}}(q, \dot{q})\right] .}
\end{aligned}
$$

The controller applied to the state dynamic equation, (11), provides the necessary value of $\ddot{p}_{a}$ to set $F_{a}$ that leads to the desired trajectory:

$$
\ddot{p}_{a}=\ddot{p}_{a}^{d}-T_{11}^{-1} T_{12} \dot{\tilde{p}}_{a}-T_{11}^{-1} \bar{M}_{e f_{a a}}^{-1}(q)\left(\bar{C}_{e f_{a a}}(q, \dot{q}) B^{T} T_{0} \tilde{x}_{e f}-\bar{u}\right) .
$$


Applying back the force transformation, (5), one obtains the value of joint torques as:

$$
\left[\begin{array}{c}
0 \\
\tau_{a}
\end{array}\right]=\left[\begin{array}{cc}
J_{u u}(q) & J_{u a}(q) \\
J_{a u}(q) & J_{a a}(q)
\end{array}\right]^{T}\left[\begin{array}{c}
F_{u} \\
F_{a}
\end{array}\right]
$$

Since $J_{u u}(q)$ is admitted invertible, $F_{u}$ is taken from the first line of (16) as $F_{u}=$ $-J_{u u}^{-T}(q) J_{a u}^{T}(q) F_{a}$, and replaced at the second line of (16), taking to

$$
\tau_{a}=\left(-J_{u a}^{T}(q) J_{u u}^{-T}(q) J_{a u}^{T}(q)+J_{a a}^{T}(q)\right) F_{a} .
$$

\section{Robust Controller Design}

\subsection{Nonlinear $\mathcal{H}_{\infty}$ Control}

The state feedback $\mathcal{H}_{\infty}$ control strategy proposed in [Chen et al. (1994)], seeks the disturbance attenuation in the system by a control law of the form $u=K(x) x$ in order to satisfy

$$
\min _{u(\cdot) \in \mathcal{L}_{2}} \max _{0 \neq \omega(\cdot) \in \mathcal{L}_{2}} \frac{\int_{0}^{\infty}\left(\frac{1}{2} \tilde{x}_{e f}^{T}(t) Q \tilde{x}_{e f}(t)+\frac{1}{2} u^{T}(t) R u(t)\right) d t}{\int_{0}^{\infty}\left(\frac{1}{2} \omega^{T}(t) \omega(t)\right) d t} \leq \gamma^{2},
$$

where $Q$ and $R$ are symmetric positive definite weighting matrices defined by the designer, $\gamma>0$ is the desired disturbance attenuation level, $\omega$ is referred to the disturbance term in (11) and $\tilde{x}_{e f}(0)=0$. Following the game theory, the known solution of this problem is given, in a simplified form, in terms of the algebraic equation

$$
\left[\begin{array}{cc}
0 & K \\
K & 0
\end{array}\right]-T_{0}^{T} B\left(R^{-1}-\frac{1}{\gamma^{2}} I\right) B^{T} T_{0}+Q=0,
$$

with $B=\left[\begin{array}{ll}I & 0\end{array}\right]^{T}$. Therefore, to solve the $\mathcal{H}_{\infty}$ problem, it is only necessary to find matrices $K$ and $T_{0}$ which solve (19). Considering the matrix $R_{1}$ the result of the Cholesky factorization

$$
R_{1}^{T} R_{1}=\left(R^{-1}-\frac{1}{\gamma^{2}} I\right)^{-1}
$$

and $Q$ factorized as

the solution of (19) is given by

$$
Q=\left[\begin{array}{cc}
Q_{1}^{T} Q_{1} & Q_{12} \\
Q_{12}^{T} & Q_{2}^{T} Q_{2}
\end{array}\right]
$$

$$
T_{0}=\left[\begin{array}{cc}
R_{1}^{T} Q_{1} & R_{1}^{T} Q_{2} \\
0 & I
\end{array}\right] \text { and } K=\frac{1}{2}\left(Q_{1}^{T} Q_{2}-Q_{2}^{T} Q_{1}\right)-\frac{1}{2}\left(Q_{21}^{T}+Q_{12}\right),
$$

with the conditions: $K>0$ and $R<\gamma^{2} I$. The optimal control input is established for the proposed application in the following.

Given a desired disturbance attenuation level $\gamma>0$, the $\mathcal{H}_{\infty}$ control problem (18) subject to (11) has an optimal solution

$$
\bar{u}^{*}=-R^{-1} B^{T} T_{0} \tilde{x}_{e f},
$$

if $\gamma^{2} I>R$ and if there exist matrices $K>0$ and a non-singular $T_{0}$ solutions of (19). The forces related to active variables can be computed applying (20) at (15), and then, using this result at (14). Joint torques are computed by (17). 
Remark 1. This nonlinear $\mathcal{H}_{\infty}$ control strategy assumes that the model structure is completely known and represents parameter uncertainties as internal disturbances, treating them the same way as external disturbances.

Remark 2. This model-based nonlinear $\mathcal{H}_{\infty}$ control strategy does not demand measured values of acceleration from the free-floating base neither from the arm.

\subsection{Adaptive Neural Network Nonlinear $\mathcal{H}_{\infty}$ Control}

Define a set of $n$ neural networks $E_{k}\left(x_{e}, \Theta_{k}\right), k=1, \cdots, n$, where $x_{e}$ is the input vector and $\Theta_{k}$ are the adjustable weights in the output layers. The single-output neural networks are of the form

$$
E_{k}\left(x_{e}, \Theta_{k}\right)=\sum_{i=1}^{p_{k}} \theta_{k i} G\left(\sum_{j=1}^{q_{k}} w_{i j}^{k} x_{e j}+b_{i}^{k}\right)=\xi_{k}^{T} \Theta_{k},
$$

where $q_{k}$ is the size of vector $x_{e}$ and $p_{k}$ is the number of neurons in the hidden layer. The weights $w_{i j}^{k}$ and the biases $b_{i}^{k}$ for $1 \leq i \leq p_{k}, 1 \leq j \leq q_{k}$ and $1 \leq k \leq n$ are assumed to be constant and specified by the designer. Thus, the adjustment of neural networks is performed only by updating the vectors $\Theta_{k}$. The activation function for the neurons in the hidden layer is chosen to be $G()=.\tanh ($.$) . The complete neural network is denoted by$

$$
E\left(x_{e}, \Theta\right)=\left[\begin{array}{c}
E_{1}\left(x_{e}, \Theta_{1}\right) \\
\vdots \\
E_{n}\left(x_{e}, \Theta_{n}\right)
\end{array}\right]=\left[\begin{array}{cccc}
\xi_{1}^{T} & 0 & \ldots & 0 \\
0 & \xi_{2}^{T} & \vdots & 0 \\
\vdots & \vdots & \ddots & \vdots \\
0 & 0 & \ldots & \xi_{n}^{T}
\end{array}\right]\left[\begin{array}{c}
\Theta_{1} \\
\Theta_{2} \\
\vdots \\
\Theta_{n}
\end{array}\right]=\Xi \Theta .
$$

Consider a first approach where the term

$$
E^{1}\left(x_{e_{e f}}, x_{e_{b}}\right)=\bar{F}\left(x_{e_{e f}}\right)+\bar{E}\left(x_{e_{b}}\right)-\delta_{a}
$$

in (11) is completely unknown regarding its structure and parameter values. The neural network defined in (22) is applied to learn the dynamic behavior of the robotic system:

$$
E^{1}\left(x_{e_{e f}}, x_{e_{b}}\right) \approx \hat{E}\left(x_{e}, \Theta\right)=\Xi \Theta,
$$

where the input vector $x_{e}$ should be defined as

$$
x_{e}=\left[\begin{array}{llllllll}
q^{T} & \dot{q}^{T} & p_{a}^{T} & \dot{p}^{T} & \ddot{p}_{u}^{T} & \left(p_{a}^{d}\right)^{T} & \left(\dot{p}_{a}^{d}\right)^{T} & \left(\ddot{p}_{a}^{d}\right)^{T}
\end{array}\right]^{T} .
$$

However, the values of $q_{b}, \dot{q}_{b}, \dot{p}_{u}$ and $\ddot{p}_{u}$ would be necessary, but they are not easy to obtain in practice. Considering that a neural network based approach is usually used when it is not possible to supply all the variables values to the system model, we have defined the vector $x_{e}$ as

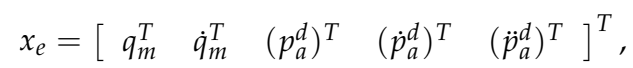

avoiding the necessity of any data from the free-floating base or related to passive variables. Simulation results will show the feasibility of this assumption. Defining the following optimization problem

$$
\Theta^{*}=\arg \min _{\Theta \in \Omega_{\Theta}} \max _{\tilde{x}_{e f} \in \Omega_{\tilde{x}_{e f}}}\left\|\hat{E}\left(x_{e}, \Theta^{*}\right)-E^{1}\left(x_{e_{e f}}, x_{e_{b}}\right)\right\|_{2^{\prime}}
$$


the modified error equation (11) may be rewritten as

$$
\begin{aligned}
\dot{\tilde{x}}_{e f} & =\bar{A}_{T_{e f}}(q, \dot{q}) \tilde{x}_{e f}+\bar{B}_{T_{e f}}(q) T_{11}\left(F_{a}-E^{1}\left(x_{e_{e f}}, x_{e_{b}}\right)+F_{d}+\hat{E}\left(x_{e}, \Theta^{*}\right)-\hat{E}\left(x_{e}, \Theta^{*}\right)\right) \\
& =\bar{A}_{T_{e f}}(q, \dot{q}) \tilde{x}_{e f}+\bar{B}_{T_{e f}}(q) T_{11}\left(F_{a}-\hat{E}\left(x_{e}, \Theta^{*}\right)\right)+\bar{B}_{T_{e f}}(q) T_{11}\left(\hat{E}\left(x_{e}, \Theta^{*}\right)-E^{1}\left(x_{e_{e f}}, x_{e_{b}}\right)+F_{d}\right) \\
& =\bar{A}_{T_{e f}}(q, \dot{q}) \tilde{x}_{e f}+\bar{B}_{T_{e f}}(q) u+\bar{B}_{T_{e f}}(q) \omega,
\end{aligned}
$$

with

$$
\begin{aligned}
u & =T_{11}\left(F_{a}-\hat{E}\left(x_{e}, \Theta^{*}\right)\right), \\
\omega & =T_{11}\left(\hat{E}\left(x_{e}, \Theta^{*}\right)-E^{1}\left(x_{e_{e f}}, x_{e_{b}}\right)+F_{d}\right),
\end{aligned}
$$

where $\omega$ refers to the estimation error from the neural network system and external disturbances. Considering $u=\bar{u}$ the control law provided by the nonlinear $\mathcal{H}_{\infty}$ controller in (20), $F_{a}$ can be computed by

$$
F_{a}=\hat{E}\left(x_{e}, \Theta^{*}\right)+T_{11}^{-1} \bar{u} .
$$

Thus, considering the stability analysis developed by [Chang \& Chen (1997)], the adaptive neural network nonlinear $\mathcal{H}_{\infty}$ control is stated for the proposed application as follows.

Let $E\left(x_{e}, \Theta\right)$ be a set of $n$ neural networks defined by (22) with $x_{e}$ being a vector of available data defined by (24) and $\Theta$ being a vector of adjustable parameters. Given a desired disturbance attenuation level $\gamma>0$ and matrices $Z=Z^{T}>0, Q=Q^{T}>0, P_{0}=P_{0}^{T}>0, Z_{0}=$ $Z_{0}^{T}>0$, and $R=R^{T}<\gamma^{2} I$, the following performance criterion

$$
\int_{0}^{T}\left(\tilde{x}_{e f}^{T} Q \tilde{x}_{e f}+\bar{u}^{T} R \bar{u}\right) d t \leq \tilde{x}_{e f}^{T}(0) P_{0} \tilde{x}_{e f}(0)+\tilde{\Theta}^{T}(0) Z_{0} \tilde{\Theta}(0)+\gamma^{2} \int_{0}^{T}\left(\omega^{T} \omega\right) d t
$$

where $\tilde{\Theta}=\Theta-\Theta^{*}$ denotes the neural parameter estimation error, is satisfied, for any initial condition, if there exists a dynamic state feedback controller

$$
\begin{aligned}
\dot{\Theta} & =\beta\left(t, \tilde{x}_{e f}\right)=-Z^{-T} \Xi^{T} T_{11} B^{T} T_{0} \tilde{x}_{e f}, \\
F_{a} & =F_{a}\left(t, \hat{\Theta}, \tilde{x}_{e f}\right)=\Xi \Theta-T_{11}^{-1} R^{-1} B^{T} T_{0} \tilde{x}_{e f},
\end{aligned}
$$

solution of the adaptive neural network nonlinear $\mathcal{H}_{\infty}$ control problem subject to (25). The torques applied upon the joints are given by (17).

On the other hand, a second approach may be proposed. Consider that model structure and nominal values for the term $\bar{F}\left(x_{e_{e f}}\right)$ are well defined and available for the controller. In this case, the neural network is applied to estimate only the behavior of parametric uncertainties and spacecraft dynamics (considered as a non-modeled dynamic):

$$
E^{2}\left(x_{e_{e f}}, x_{e_{b}}\right) \approx \hat{E}\left(x_{e}, \Theta\right)=\Xi \Theta
$$

where $E^{2}\left(x_{e_{e f}}, x_{e_{b}}\right)=\bar{E}\left(x_{e_{b}}\right)-\delta_{a}$.

Similarly, $x_{e}$ is defined by (24), the optimal approximation parameters vector is given by

$$
\Theta^{*}=\arg \min _{\Theta \in \Omega_{\Theta}} \max _{\tilde{x}_{e f} \in \Omega_{\tilde{x}_{e f}}}\left\|\hat{E}\left(x_{e}, \Theta^{*}\right)-E^{2}\left(x_{e_{e f}}, x_{e_{b}}\right)\right\|_{2^{\prime}}
$$


and the modified error equation (11) may be rewritten as

$$
\begin{aligned}
\dot{\tilde{x}}_{e f} & =\bar{A}_{T_{e f}}(q, \dot{q}) \tilde{x}_{e f}+\bar{B}_{T_{e f}}(q) T_{11}\left(F_{a}-\bar{F}\left(x_{e_{e f}}\right)-E^{2}\left(x_{e_{e f}}, x_{e_{b}}\right)+F_{d}+\hat{E}\left(x_{e}, \Theta^{*}\right)-\hat{E}\left(x_{e}, \Theta^{*}\right)\right) \\
& =\bar{A}_{T_{e f}}(q, \dot{q}) \tilde{x}_{e f}+\bar{B}_{T_{e f}}(q) T_{11}\left(F_{a}-\bar{F}\left(x_{e_{e f}}\right)-\hat{E}\left(x_{e}, \Theta^{*}\right)\right)+\bar{B}_{T_{e f}}(q) T_{11}\left(\hat{E}\left(x_{e}, \Theta^{*}\right)+\right. \\
& \left.-E^{2}\left(x_{e_{e f}}, x_{e_{b}}\right)+F_{d}\right) \\
& =\bar{A}_{T_{e f}}(q, \dot{q}) \tilde{x}_{e f}+\bar{B}_{T_{e f}}(q) u+\bar{B}_{T_{e f}}(q) \omega,
\end{aligned}
$$

with

$$
\begin{aligned}
u & =T_{11}\left(F_{a}-\bar{F}\left(x_{e_{e f}}\right)-\hat{E}\left(x_{e}, \Theta^{*}\right)\right), \\
\omega & =T_{11}\left(\hat{E}\left(x_{e}, \Theta^{*}\right)-E^{2}\left(x_{e_{e f}}, x_{e_{b}}\right)+F_{d}\right),
\end{aligned}
$$

where $\omega$ refers to the estimation error from the neural network system and external disturbances. Considering $u=\bar{u}$ the control law provided by the nonlinear $\mathcal{H}_{\infty}$ controller in (20), $F_{a}$ can be computed by

$$
F_{a}=\bar{F}\left(x_{e_{e f}}\right)+\hat{E}\left(x_{e}, \Theta^{*}\right)+T_{11}^{-1} \bar{u} .
$$

For this approach, the adaptive neural network nonlinear $\mathcal{H}_{\infty}$ control can be enunciated as follows.

Let $E\left(x_{e}, \Theta\right)$ be a set of $n$ neural networks defined by (22) with $x_{e}$ being a vector of available data defined by (24) and $\Theta$ being a vector of adjustable parameters. Given a desired disturbance attenuation level $\gamma>0$ and matrices $Z=Z^{T}>0, Q=Q^{T}>0, P_{0}=P_{0}^{T}>0, Z_{0}=$ $Z_{0}^{T}>0$, and $R=R^{T}<\gamma^{2} I$, the following performance criterion

$$
\int_{0}^{T}\left(\tilde{x}_{e f}^{T} Q \tilde{x}_{e f}+\bar{u}^{T} R \bar{u}\right) d t \leq \tilde{x}_{e f}^{T}(0) P_{0} \tilde{x}_{e f}(0)+\tilde{\Theta}^{T}(0) Z_{0} \tilde{\Theta}(0)+\gamma^{2} \int_{0}^{T}\left(\omega^{T} \omega\right) d t
$$

where $\tilde{\Theta}=\Theta-\Theta^{*}$ denotes the neural parameter estimation error, is satisfied, for any initial condition, if there exists a dynamic state feedback controller

$$
\begin{aligned}
& \dot{\Theta}=\beta\left(t, \tilde{x}_{e f}\right)=-Z^{-T} \Xi^{T} T_{11} B^{T} T_{0} \tilde{x}_{e f}, \\
& F_{a}=F_{a}\left(t, \hat{\Theta}, \tilde{x}_{e f}\right)=\bar{F}\left(x_{e_{e f}}\right)+\Xi \Theta-T_{11}^{-1} R^{-1} B^{T} T_{0} \tilde{x}_{e f},
\end{aligned}
$$

solution of the adaptive neural network nonlinear $\mathcal{H}_{\infty}$ control problem subject to (33). The stability analysis developed in [Chang \& Chen (1997)] is also valid for this case, [Petronilho et al. (2005)]. The torques applied upon the joints are given by (17).

Remark 3. The adaptive neural network nonlinear $\mathcal{H}_{\infty}$ strategies do not demand measured values of acceleration from the free-floating base neither from the arm.

Remark 4. The adaptive designs proposed apply an intelligent learning strategy to estimate uncertain parameters and also the behavior of non-modeled dynamics. The $\mathcal{H}_{\infty}$ control law is applied to attenuate the effects of estimation errors and external disturbances. 


\section{Results}

For validation and comparison purposes, the proposed adaptive $\mathcal{H}_{\infty}$ control solutions are applied to a free-floating, planar, two-link space manipulator system, whose nominal parameters are given in Table 1 . The corresponding DEM is a fixed-base, three-link, planar manipulator whose first joint is configured as passive, that is, $q_{m}=\left[\begin{array}{ll}q_{2} & q_{3}\end{array}\right]^{T}$ are the joints to be controlled. Its structure is based on the fixed-base manipulator UArmII (Underactuated Arm II), whose nominal parameters are given in Table 2. Nominal matrices $M(q)$ and $C(q, \dot{q})$ for the DEM can be found in [Liang et al. (1996)] and the Jacobian is given in the appendix.

\begin{tabular}{ccccc}
\hline \hline Body & $m_{i}{ }^{\prime}$ & $I_{i}{ }^{\prime}$ & $R_{i}$ & $L_{i}$ \\
\hline & $(\mathrm{kg})$ & $\left(\mathrm{kgm}^{2}\right)$ & $(m)$ & $(\mathrm{m})$ \\
\cline { 2 - 5 } Base & 4.816 & 0.008251 & 0.253 & 0 \\
Link 2 & 0.618 & 0.0075 & 0.118 & 0.12 \\
Link 3 & 0.566 & 0.006 & 0.126 & 0.085 \\
\hline \hline
\end{tabular}

Table 1. SM Parameters

\begin{tabular}{ccccc}
\hline \hline Body & $m_{i}$ & $I_{i}$ & $W_{i}$ & $l c_{i}$ \\
\hline & $(k g)$ & $\left(\mathrm{kgm}^{2}\right)$ & $(m)$ & $(m)$ \\
\cline { 2 - 5 } Link 1 & 1.932 & 0.008251 & 0.203 & 0 \\
Link 2 & 0.850 & 0.0075 & 0.203 & 0.096 \\
Link 3 & 0.625 & 0.006 & 0.203 & 0.077 \\
\hline \hline
\end{tabular}

Table 2. DEM Parameters

A trajectory tracking task is defined for the space manipulator end-effector. The Cartesian positions $p_{a}=\left[\begin{array}{ll}x_{e f} & y_{e f}\end{array}\right]^{T}$ of the end-effector are chosen to be the controlled variables, while its orientation $\phi_{e f}$ is let free. The reference trajectory is defined as a semi-circle starting at the end-effector initial position (set by $q(0)=\left[\begin{array}{lll}0^{\circ} & 20^{\circ} & -40^{\circ}\end{array}\right]^{T}$ ) and characterized by radius $=5 \mathrm{~cm}$. The angles that determine the semi-circle reference trajectory follows a fifth degree polynomial with $t_{f}=3 s$ (time defined for the task execution). During the simulation, a limited disturbance, initializing at $t=1 \mathrm{~s}$, was introduced in the following form

$$
\tau_{d}=\left[\begin{array}{l}
0.025 e^{-2 t} \sin (2 \pi t) \\
0.015 e^{-2 t} \sin (2 \pi t)
\end{array}\right]
$$

Compared to the torque applied in case that none disturbance is inserted, the disturbance $\tau_{d}$ presents peaks of approximately $75 \%$ of that torque peak value. Multiplicative uncertainties were also applied to the values of mass, moment of inertia, length and center of mass position as $\delta=\left[\begin{array}{llll}0.7 * m & 1.2 * I & 1.7 * W & 0.5 * l_{c}\end{array}\right]$.

In order to clearly identify the controllers actuation, Figures 2 to 5 illustrate the results obtained without adding disturbances and uncertainties to the model (nominal case) while Figures 6 to 9 show the results for the disturbed situation (disturbed case). To establish a basis for comparison, define a computed torque control by

$$
\ddot{p}_{a}=K_{p} \tilde{p}_{a}+K_{d} \dot{\tilde{p}}_{a}+K_{i} \int \tilde{p}_{a}+\ddot{p}_{a}^{d},
$$

with $K_{p}=50 I_{2}, K_{d}=\left[\begin{array}{cc}50 \sqrt{\left|K_{p}\right|} & 0 \\ 0 & 1.5 \sqrt{\left|K_{p}\right|}\end{array}\right]$ and $K_{i}=I_{2}$. Applying (40) to (14) provides the forces related to active variables and joint torques are computed by (17). Simulation results are presented by Figures 2 and 6 .

The level of disturbance attenuation defined for the proposed nonlinear $\mathcal{H}_{\infty}$ controllers is $\gamma=2$. The selected weighting matrices are shown in Table 3. Figures 3 and 7 are the results obtained by the nonlinear $\mathcal{H}_{\infty}$ control described in Section 3.1. For the nonlinear $\mathcal{H}_{\infty}$ controllers via neural network proposed, let $n=2$ be the size of $p_{a}$ determined by the number 
of joints of the space manipulator (active joints in DEM), which define the size of $x_{e}, q_{k}=10$. Define $E\left(x_{e}, \Theta\right):=\left[\begin{array}{lll}E_{1}\left(x_{e}, \Theta_{1}\right) & E_{2}\left(x_{e}, \Theta_{2}\right)\end{array}\right]^{T}$ with $p_{k}=7$ neurons in the hidden layer, the bias vector $b_{k}=\left[\begin{array}{lllllll}-3 & -2 & -1 & 0 & 1 & 2 & 3\end{array}\right]$ and the weighting matrix for the first layer

$$
\Omega_{i}^{k}=\left[\omega_{i j}^{k}\right]=\left[\begin{array}{llllllllll}
1 & 1 & 1 & 1 & -1 & -1 & -1 & -1 & -1 & -1
\end{array}\right] .
$$

The uncertain vector $\Theta$ is defined as $\Theta=\left[\begin{array}{ll}\Theta_{1} & \Theta_{2}\end{array}\right]^{T}$, with $\Theta_{1}^{T}=\left[\begin{array}{lll}\theta_{11} & \cdots & \theta_{17}\end{array}\right]$ and $\Theta_{2}^{T}=\left[\begin{array}{lll}\theta_{21} & \cdots & \theta_{27}\end{array}\right]$, and the matrix $\Xi$ can be computed with $\xi_{1}^{T}=\left[\begin{array}{lll}\xi_{11} & \cdots & \xi_{17}\end{array}\right]$ and $\xi_{2}^{T}=\left[\begin{array}{lll}\xi_{21} & \cdots & \xi_{27}\end{array}\right]$. Simulation results for the adaptive neural network nonlinear $\mathcal{H}_{\infty}$ controller are shown in Figures 4 and 8 . For the second approach, neural network plus nominal model, simulation results are shown in Figures 5 and 9.

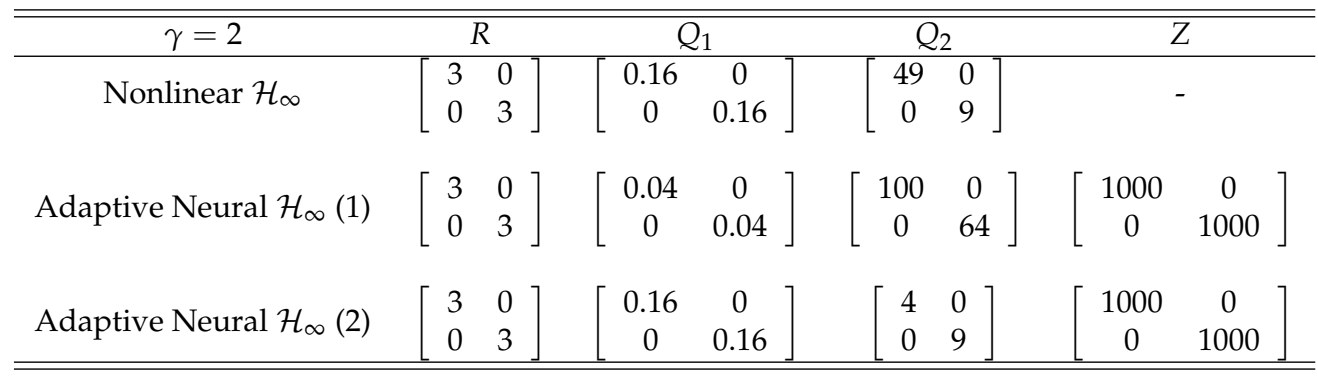

Table 3. Selected Weighting Matrices
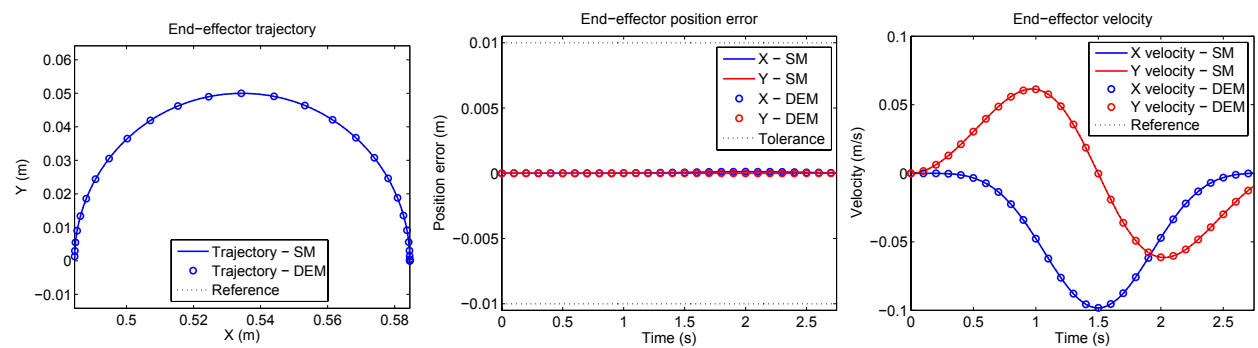

Fig. 2. Nominal case: End-effector trajectory, position errors and velocities - Computed torque
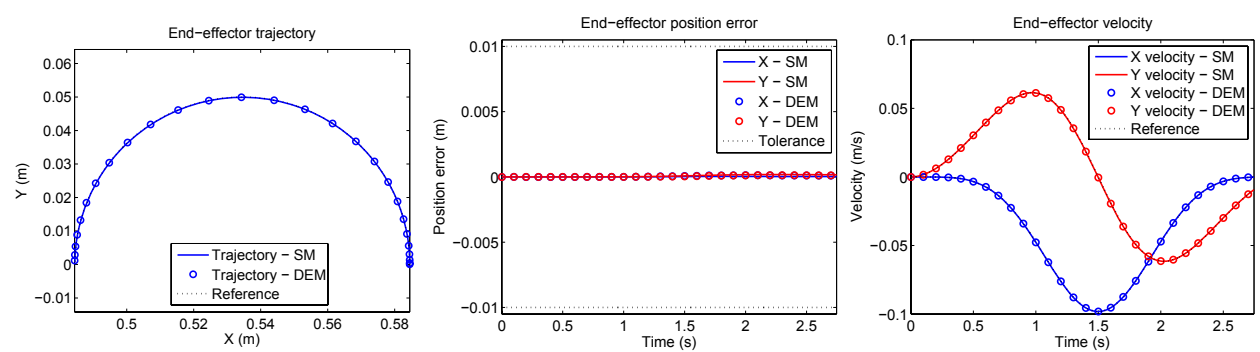

Fig. 3. Nominal case: End-effector trajectory, position errors and velocities - Nonlinear $\mathcal{H}_{\infty}$ 

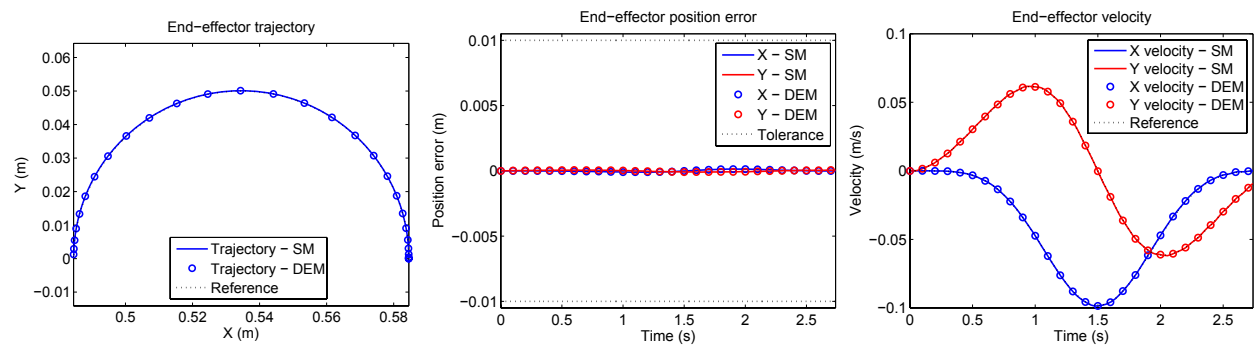

Fig. 4. Nominal case: End-effector trajectory, position errors and velocities - Adaptive Neural Network Nonlinear $\mathcal{H}_{\infty}(1)$
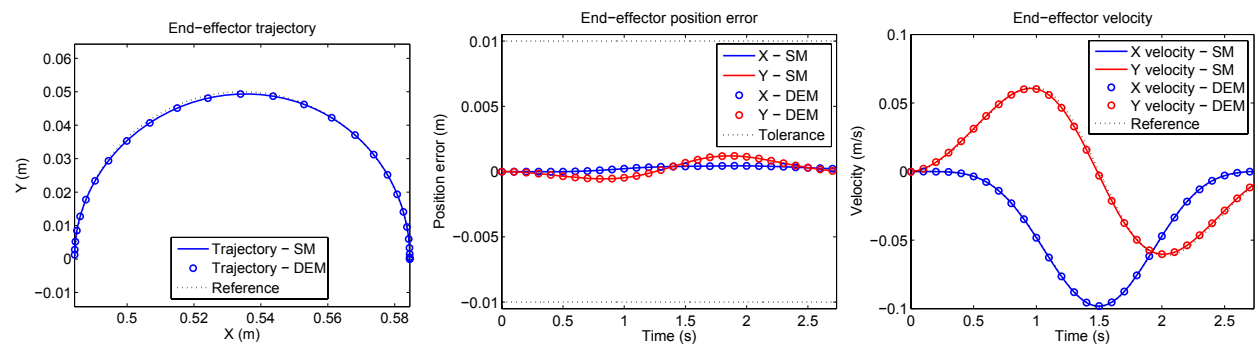

Fig. 5. Nominal case: End-effector trajectory, position errors and velocities - Adaptive Neural Network Nonlinear $\mathcal{H}_{\infty}(2)$
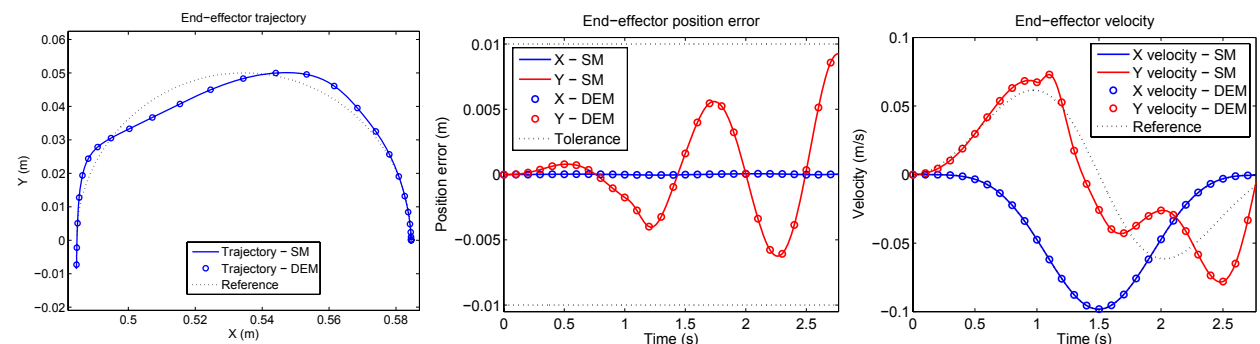

Fig. 6. Disturbed case: End-effector trajectory, position errors and velocities - Computed torque
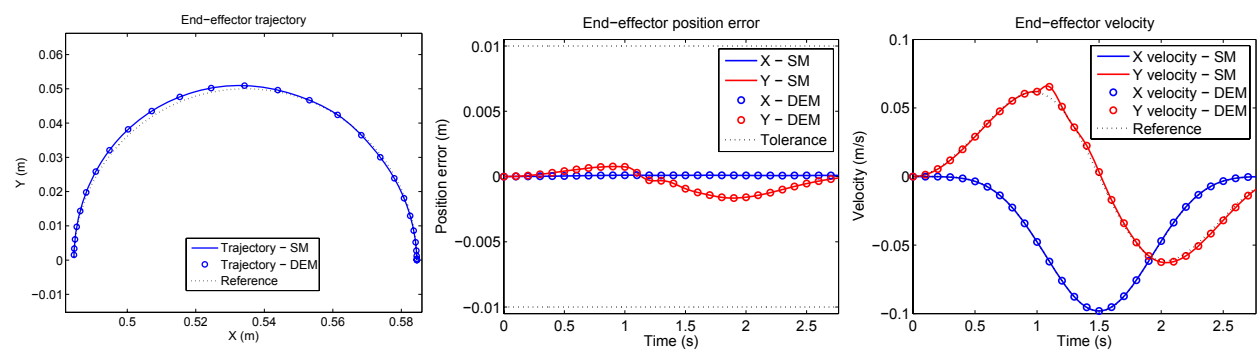

Fig. 7. Disturbed case: End-effector trajectory, position errors and velocities - Nonlinear $\mathcal{H}_{\infty}$ 

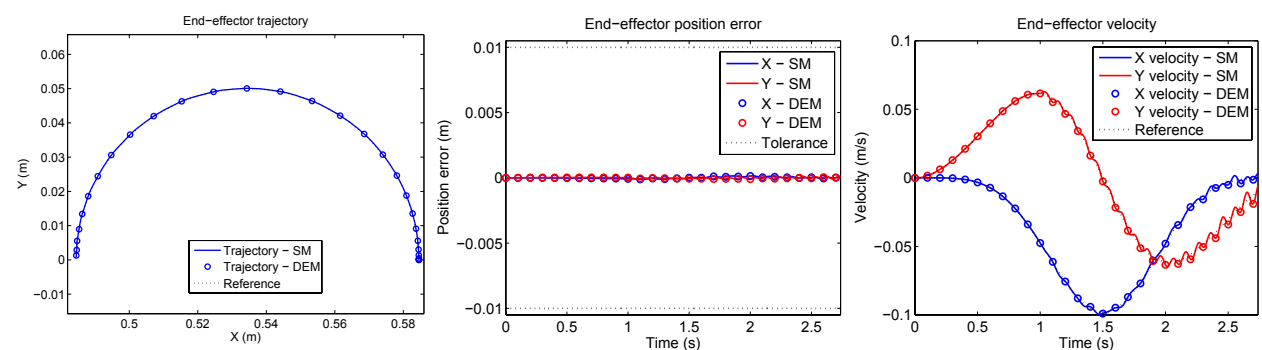

Fig. 8. Disturbed case: End-effector trajectory, position errors and velocities - Adaptive Neural Network Nonlinear $\mathcal{H}_{\infty}(1)$
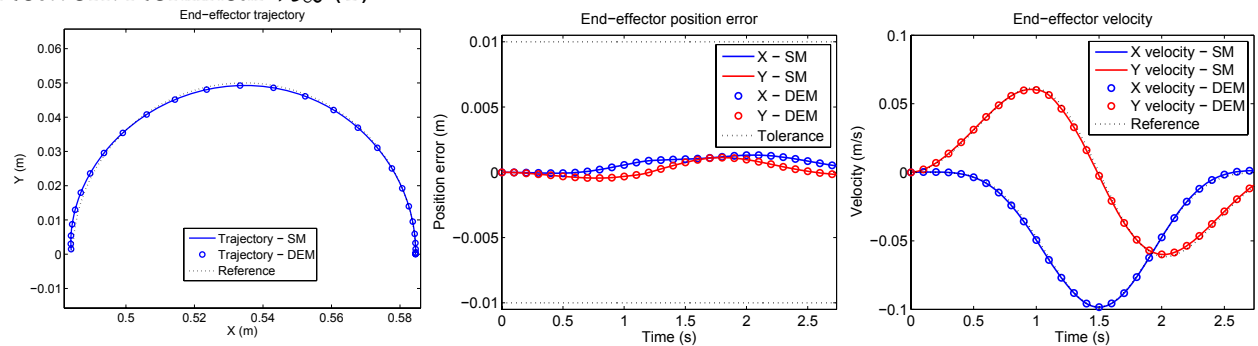

Fig. 9. Disturbed case: End-effector trajectory, position errors and velocities - Adaptive Neural Network Nonlinear $\mathcal{H}_{\infty}(2)$

A comparison among the graphical results illustrate that the proposed controllers reject disturbance efficiently and attenuate its effect in the trajectory tracking task. The computed torque control, however, presents an excellent result in Figure 2, where the plant model is completely defined and no disturbances and uncertainties are applied, but it is not able to reject the disturbances and uncertainties effects when they occur, Figure 6 . These results clearly demonstrate the robustness of $\mathcal{H}_{\infty}$ criterion.

Considering that the same value of $\gamma$ was applied to all the proposed controllers, two performance indexes are used to numerically compare the controllers applied: the $\mathcal{L}_{2}$ norm of the state vector

$$
\mathcal{L}_{2}\left[\tilde{x}_{e f}\right]=\left(\frac{1}{\left(t_{r}-t_{0}\right)} \int_{t_{0}}^{t_{r}}\left\|\tilde{x}_{e f}(t)\right\|_{2}^{2} d t\right)^{1 / 2},
$$

where $\|\cdot\|_{2}$ is the Euclidean norm, and the sum of the applied torques

$$
E\left[\tau_{a}\right]=\sum_{i=1}^{2}\left(\int_{t_{0}}^{t_{r}}\left|\tau_{a_{i}}(t)\right| d t\right) .
$$

The results are presented in Table 4 as follows. A quantitative analysis based on Table 4 endorses the results verified by the graphics. The robustness provided by the nonlinear $\mathcal{H}_{\infty}$ control law is shown by the $\mathcal{L}_{2}\left[\tilde{x}_{e f}\right]$ results when compared to the results presented by the computed torque method. The results presented by the adaptive neural networks approaches exhibits their efficiency in estimating the effect of uncertainties, and mainly, the non-modeled dynamics of the spacecraft. The energy spent by the first approach of adaptive neural network strategy is greater than the one spent by strategy (2), however, their error avoidance capacity 


\begin{tabular}{ccccc}
\hline \hline & \multicolumn{2}{c}{ Nominal case } & \multicolumn{2}{c}{ Disturbed case } \\
\cline { 2 - 5 } & $\mathcal{L}_{2}\left[\tilde{x}_{e f}\right]$ & $E\left[\tau_{a}\right](\mathrm{Nms})$ & $\mathcal{L}_{2}\left[\tilde{x}_{e f}\right]$ & $E\left[\tau_{a}\right](\mathrm{Nms})$ \\
\cline { 2 - 5 } Computed Torque & $1.24 \times 10^{-4}$ & 0.0449 & 0.0210 & 0.0592 \\
Nonlinear $\mathcal{H}_{\infty}$ & $1.39 \times 10^{-4}$ & 0.0449 & 0.0024 & 0.0458 \\
Adaptive Neural $\mathcal{H}_{\infty}(1)$ & $3.63 \times 10^{-4}$ & 0.0452 & 0.0022 & 0.0559 \\
Adaptive Neural $\mathcal{H}_{\infty}(2)$ & $16 \times 10^{-4}$ & 0.0442 & 0.0020 & 0.0444 \\
\hline \hline
\end{tabular}

Table 4. Performance Indexes

are very similar. Thus, the designer should ponder between energy demand and availability of the plant model. It must be emphasized that none of the proposed $\mathcal{H}_{\infty}$ approaches demands measured acceleration values from the free-floating base or the arm.

\section{Conclusion}

This chapter presents an investigation on the motion control of a free-floating space manipulator subject to parametric uncertainties and external disturbances performed by different methods of nonlinear $\mathcal{H}_{\infty}$ controllers.

The free-floating space manipulator model is developed based on the Dynamically Equivalent Manipulator concept. Trajectory tracking of the SM's end-effector in task (Cartesian) space is considered including the existence of parametric uncertainties in the model, the application of external disturbances and considering unknown the spacecraft dynamics. Nonlinear $\mathcal{H}_{\infty}$ control techniques are designed according to the knowledge and availability of the parameter matrices for the controllers. Simulations in a free-floating space planar manipulator with two links were evaluated to demonstrate the effectiveness of the presented approaches. A qualitative analysis of trajectory tracking graphics identifies the action of the proposed control laws. A quantitative comparison among the strategies is provided by performance indexes regarding energy consumption and error avoidance.

The nonlinear $\mathcal{H}_{\infty}$ controller procedure demands a precise knowledge of the model structure, but its efficiency in attenuating the effects of parametric uncertainties and external disturbances was verified before the results of a computed torque controller. The first approach for adaptive neural network $\mathcal{H}_{\infty}$ controller applies the intelligent system to learn the dynamic behavior of the robotic system, which is considered totally unknown. This strategy proved to be very effective in estimating the non-modeled plant behavior. The proposal of combining the model-based technique (once the model is largely known) and the intelligent adaptive tool, joined the best characteristics of both strategies, robustness and flexibility, and exhibited the best numerical results.

The space applications area of research is wide and full of unique issues. Future research on the topic presented in this chapter should include application of the proposed strategies in a real robot arm, supported by the modeling concept used in this chapter, and also the proposal of other intelligent techniques, such as fuzzy systems.

\section{References}

Abiko, S. \& Hirzinger, G. (2009). Frontiers in Adaptive Control, IN-TECH, chapter An Adaptive Control for a Free-Floating Space Robot by Using Inverted Chain Approach, pp. 1-16. 
Caccavale, F. \& Siciliano, B. (2001). Quaternion-based kinematic control of redundant spacecraft/manipulator systems, IEEE International Conference on Robotics and Automation (ICRA), Vol. 1, Seoul, Korea, pp. 435-440. doi:10.1109/ROBOT.2001.932589.

Chang, Y. C. \& Chen, B. S. (1997). A nonlinear adaptive $\mathcal{H}_{\infty}$ tracking control design in robotic systems via neural networks, IEEE Transactions on Control Systems Technology 5(1): 1329. doi:10.1109/87.553662.

Chen, B. S., Lee, T. S. \& Feng, J. H. (1994). A nonlinear $\mathcal{H}_{\infty}$ control design in robotic systems under parameter perturbation and external disturbance, International Journal of Control 59(2): 439-461. doi:10.1080/00207179408923085.

Dubowsky, S. \& Papadopoulos, E. (1993). The kinematics, dynamics, and control of free-flying and free-floating space robotic systems, IEEE Transactions on Robotics and Automation 9(5): 531-543. doi:10.1109/70.258046.

Dubowsky, S. \& Torres, M. A. (1991). Path planning for space manipulators to minimize spacecraft attitude disturbances, IEEE International Conference on Robotics and Automation (ICRA), Vol. 3, Sacramento, USA, pp. 2522-2528. doi:10.1109/ROBOT.1991.132005.

Fu, Y., Zhang, F., Wang, S. \& Chi, Z. (2007). A dynamic control method for free-floating space manipulator in task space, IEEE International Conference on Robotics and Biomimetics, Sanya, China, pp. 1230-1235. doi:.

$\mathrm{Gu}$, Y. L. \& Xu, Y. (1993). A normal form augmentation approach to adaptive control of space robot systems, IEEE International Conference on Robotics and Automation (ICRA), Vol. 2, Atlanta, USA, pp. 731-737. doi:10.1109/ROBOT.1993.291872.

Huang, P. \& Xu, Y. (2006). Pso-based time-optimal trajectory planning for space robot with dynamic constraints, IEEE International Conference on Robotics and Biomimetics (ROBIO), Kunming, China, pp. 1402-1407. doi:10.1109/ROBIO.2006.340134.

Johansson, R. (1990). Quadratic optimization of motion coordination and control, IEEE Transactions on Automatic Control 35(11): 1197-1208. doi:10.1109/9.59805.

Lewis, F. L., Abdallah, C. T. \& Dawson, D. M. (1993). Control of Robot Manipulators, Macmillan Publishing Company, New York.

Liang, B., Xu, Y. \& Bergerman, M. (1996). Mapping a space manipulator to a dynamically equivalent manipulator, Technical Report CMU-RI-TR-96-33, Robotics Institute, Carnegie Mellon University, Pittsburgh, PA. doi:10.1.1.67.6435.

Liu, Z., Huang, P., Yan, J. \& Liu, G. (2009). Multi-objective genetic algorithms for trajectory optimization of space manipulator, IEEE Conference on Industrial Electronics and Applications (ICIEA), 4th, Xi'an, China, pp. 2810-2815. doi:10.1109/ICIEA.2009.5138722.

Papadopoulos, E. \& Dubowsky, S. (1991). On the nature of control algorithms for freefloating space manipulators, IEEE Transactions on Robotics and Automation 7(6): 750758. doi:10.1109/70.105384.

Papadopoulos, E., Tortopidis, I. \& Nanos, K. (2005). Smooth planning for free-floating space robots using polynomials, IEEE International Conference on Robotics and Automation (ICRA), Barcelona, Spain, pp. 4272-4277.

Parlaktuna, O. \& Ozkan, M. (2004). Adaptive control of free-floating space robots in cartesian coordinates, Advanced Robotics 18(9): 943-959. doi:10.1163/1568553042225732.

Petronilho, A., Siqueira, A. A. G. \& Terra, M. H. (2005). Adaptive $\mathcal{H}_{\infty}$ tracking control design via neural networks of a constrained robot system, IEEE Conference on Decision and Control, 44th, Seville, Spain, pp. 5528-5533. 
Siqueira, A. A. G. \& Terra, M. H. (2004). A fault tolerant robot manipulator based on $\mathcal{H}_{2}$, $\mathcal{H}_{\infty}$ and mixed $\mathcal{H}_{2} / \mathcal{H}_{\infty}$ markovian controls, IEEE Conference on Control Applications (CCA), Taipei, Taiwan.

Taveira, T. F. P. A., Siqueira, A. A. G. \& Terra, M. H. (2006). Adaptive nonlinear $\mathcal{H}_{\infty}$ controllers applied to a free-floating space manipulator, IEEE International Conference on Control Applications (CCA), Munich, Germany, pp. 1476-1481. doi:10.1109/CCA.2006.286094.

Torres, M. A. \& Dubowsky, S. (1992). Minimizing spacecraft attitude disturbances in space manipulators systems, AIAA Journal of Guidance, Control, and Dynamics 15(4): 10101017. doi:10.2514/3.20936.

Tortopidis, I. \& Papadopoulos, E. (2006). Point-to-point planning: Methodologies for underactuated space robots, IEEE International Conference on Robotics and Automation (ICRA), Orlando, USA, pp. 3861-3866. doi:10.1109/ROBOT.2006.1642293.

\section{A. Appendix}

Let the auxiliary variables be:

$$
\begin{aligned}
& r_{1}=\frac{R_{1} m_{1}}{M t}, \\
& r_{2}=\frac{R_{2}\left(m_{1}+m_{2}\right)}{M t}, \\
& r_{3}=\frac{R_{3}\left(m_{1}+m_{2}+m_{3}\right)}{M t}, \\
& l_{2}=\frac{L_{2} m_{1}}{M t}, \\
& l_{3}=\frac{L_{3}\left(m_{1}+m_{2}\right)}{M t} .
\end{aligned}
$$

The Jacobian is given by

$$
\begin{gathered}
J(q)=\left[\begin{array}{lll}
J_{11}(q) & J_{12}(q) & J_{13}(q) \\
J_{21}(q) & J_{22}(q) & J_{23}(q) \\
J_{31}(q) & J_{32}(q) & J_{33}(q)
\end{array}\right] \\
J_{11}(q)=J_{12}(q)=J_{13}(q)=1 \\
J_{21}(q)=-r_{1} \sin \left(q_{1}\right)-\left(r_{2}+l_{2}\right) \sin \left(q_{1}+q_{2}\right)-\left(r_{3}+l_{3}\right) \sin \left(q_{1}+q_{2}+q_{3}\right), \\
J_{22}(q)=-\left(r_{2}+l_{2}\right) \sin \left(q_{1}+q_{2}\right)-\left(r_{3}+l_{3}\right) \sin \left(q_{1}+q_{2}+q_{3}\right) \\
J_{23}(q)=-\left(r_{3}+l_{3}\right) \sin \left(q_{1}+q_{2}+q_{3}\right) \\
J_{31}(q)=r_{1} \cos \left(q_{1}\right)+\left(r_{2}+l_{2}\right) \cos \left(q_{1}+q_{2}\right)+\left(r_{3}+l_{3}\right) \cos \left(q_{1}+q_{2}+q_{3}\right), \\
J_{32}(q)=\left(r_{2}+l_{2}\right) \cos \left(q_{1}+q_{2}\right)+\left(r_{3}+l_{3}\right) \cos \left(q_{1}+q_{2}+q_{3}\right) \\
J_{33}(q)=\left(r_{3}+l_{3}\right) \cos \left(q_{1}+q_{2}+q_{3}\right) .
\end{gathered}
$$




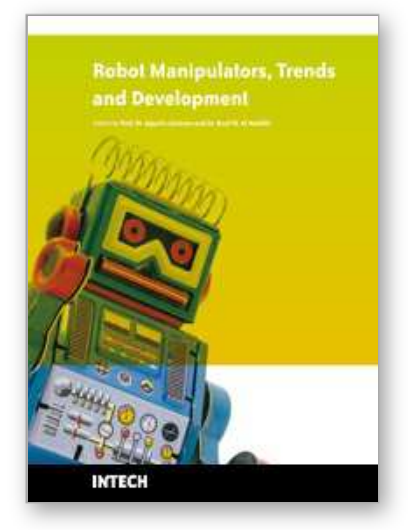

\author{
Robot Manipulators Trends and Development \\ Edited by Agustin Jimenez and Basil M Al Hadithi
}

ISBN 978-953-307-073-5

Hard cover, 666 pages

Publisher InTech

Published online 01, March, 2010

Published in print edition March, 2010

This book presents the most recent research advances in robot manipulators. It offers a complete survey to the kinematic and dynamic modelling, simulation, computer vision, software engineering, optimization and design of control algorithms applied for robotic systems. It is devoted for a large scale of applications, such as manufacturing, manipulation, medicine and automation. Several control methods are included such as optimal, adaptive, robust, force, fuzzy and neural network control strategies. The trajectory planning is discussed in details for point-to-point and path motions control. The results in obtained in this book are expected to be of great interest for researchers, engineers, scientists and students, in engineering studies and industrial sectors related to robot modelling, design, control, and application. The book also details theoretical, mathematical and practical requirements for mathematicians and control engineers. It surveys recent techniques in modelling, computer simulation and implementation of advanced and intelligent controllers.

\title{
How to reference
}

In order to correctly reference this scholarly work, feel free to copy and paste the following:

Tatiana Pazelli, Marco Terra and Adriano Siqueira (2010). Adaptive Robust Controller Designs Applied to Free-Floating Space Manipulators in Task Space, Robot Manipulators Trends and Development, Agustin Jimenez and Basil M Al Hadithi (Ed.), ISBN: 978-953-307-073-5, InTech, Available from:

http://www.intechopen.com/books/robot-manipulators-trends-and-development/adaptive-robust-controllerdesigns-applied-to-free-floating-space-manipulators-in-task-space

\section{INTECH}

open science | open minds

\section{InTech Europe}

University Campus STeP Ri

Slavka Krautzeka 83/A

51000 Rijeka, Croatia

Phone: +385 (51) 770447

Fax: +385 (51) 686166

www.intechopen.com

\section{InTech China}

Unit 405, Office Block, Hotel Equatorial Shanghai

No.65, Yan An Road (West), Shanghai, 200040, China

中国上海市延安西路65号上海国际贵都大饭店办公楼 405 单元

Phone: +86-21-62489820

Fax: $+86-21-62489821$ 
(C) 2010 The Author(s). Licensee IntechOpen. This chapter is distributed under the terms of the Creative Commons Attribution-NonCommercialShareAlike-3.0 License, which permits use, distribution and reproduction for non-commercial purposes, provided the original is properly cited and derivative works building on this content are distributed under the same license. 